\title{
Phasing ALMA with the 64-antenna Correlator
}

\author{
Alain Baudry ${ }^{1}$ \\ Univ. Bordeaux, LAB, UMR5804, F-33270 Floirac, France \\ CNRS, LAB, UMR5804, F-33270 Floirac, France \\ \& European ALMA Project Office, ESO, K. Schwarzschild Str. 2, 85748 Garching, Germany \\ E-mail: baudrylobs.u-bordeauxl. fr \\ Richard Lacasse, Ray Escoffier, Joe Greenberg, Robert Treacy \\ National Radio Astronomy Observatory \\ NRAO Technology Center, 1180 Boxwood Estate Road, Charlottesville, VA 22903-4608, USA \\ E-mail: rlacasseenrao.edu
}

\author{
Alejandro Saez \\ Joint ALMA Office \\ Alonso de Cordova 3107, Vitacura, Santiago de Chile, Chile \\ E-mail: asaezenrao.edu
}

\begin{abstract}
The main characteristics of the ALMA 64-antenna correlator and its status are very briefly presented. It is recalled that the basic elements required to phase up the ALMA array, and thus enhance the ALMA science objectives, have been included in the correlator original design. We give an overview of the new phasing interface card which needs to be added to the 64-antenna correlator to implement the VLBI mode in ALMA. Finally, we briefly describe the test fixture which is planned to validate in the laboratory the new phasing card and the VLBI mode without disturbing ALMA current operation.
\end{abstract}

11th European VLBI Network Symposium \& Users Meeting

October 9-12, 2012

Bordeaux, France

${ }^{1}$ Speaker 


\section{Introduction}

The Atacama Large Millimeter/submillimeter Array (ALMA) is the largest ground-based international astronomy facility ever built. ALMA consists of 66 antennas deployed on the desertic Atacama plateau in northern Chile. The antennas are equipped with 10 frequency bands spanning the range 31 to $950 \mathrm{GHz}$ (bands 1 and 2 are still under development). They can be relocated on fixed antenna stations for optimum radio imaging synthesis (see e.g. [1]). In its current 'connected array' configuration, where antennas are separated from a few tens of meters to several kilometers, ALMA provides highly sensitive and high spatial resolution maps in both narrow and broad spectral channels.

Another observing mode will consist of phasing the ALMA antennas to combine the large ALMA collecting area with other mm- or submm-wave antennas distributed across several continents to perform VLBI observations. The ALMA telescope has the potential to be the most sensitive element of a world-wide (sub)mm VLBI array. Hooks were included in the ALMA 64antenna correlator to facilitate phasing ALMA. This possibility is being used by a group of engineers and scientists interested in setting up a sensitive array to image the black hole at the center of our own Galaxy with 'event horizon' spatial scale resolution (e.g. [2]). Phasing ALMA would also enable addressing a broad range of topics in addition to the event horizon project (e.g. [2], [3]). In this contribution, we describe the main properties of the ALMA correlator and the design of a new interface card and test fixture required for the phasing project.

\section{The 64-antenna correlator}

\subsection{Specifications and FXF architecture}

The 64-antenna correlator is a digital hybrid correlator (FXF) which processes up to 64 antennas at $16 \mathrm{GHz}$ bandwidth in 4 basebands for each of two different polarizations (Table 1).

Table 1. Top level specifications of the ALMA main array correlator.

Item

Antennas

Baseband inputs per antenna

Input sample format

Output correlation sample format

Processing rate

Spectral points per baseband (Frequency Division Mode)

Spectral points per baseband (Time Division Mode)

Polarization products

\section{Specification}

$\leq 64$

$8 \times 2 \mathrm{GHz}$

3-bit, 8-level at 4 Gsample/s

2-bit, 4-level or 4-bit, 16-level

$125 \mathrm{MHz}$

$\leq 8192$ per baseband

64,128 or 256

1,2 or 4

A very brief description of some main elements of the ALMA 64-antenna correlator is given below; the emphasis is on the elements most relevant to phasing ALMA. More details on this correlator and the companion ALMA compact array correlator processing the 16 antennas of the compact aray can be found in [4].

The input stage of the 64-antenna correlator is the Tunable Filter Bank (TFB), a digital filter sub-system which divides the $2 \mathrm{GHz}$ input baseband into 32 sub-bands of $62.5 \mathrm{MHz}$. The 
center frequency of each sub-band can be placed anywhere within the $2 \mathrm{GHz}$ baseband. The 32 frequency-mobile digital filters are implemented in 16 large Field Programmable Gate Arrays assembled on a single card. As in a system with a pure FX architecture, the number of filters increases in proportion with the number of antennas; since there are 8 TFB cards per antenna for the 4 baseband pairs, the total number of TFB cards required is $8 \times 64=512$.

One of the basic elements of the correlator system is the 'correlator plane', a matrix of $64 \mathrm{x}$ 64 correlation 'blocks' with 256-lag correlator programmable circuits at each intersection of the matrix. When the TFB cards are by-passed the FXF system is equivalent to a pure X-lag correlator. Because the correlator clock is $1 / 32$ of the $4 \mathrm{GHz}$ sampling rate there are 32 planes per baseband pair, each one processing $1 \mathrm{msec}$ time bins of the sampled data for all antennas. The outputs from these 32 planes are summed to keep up with the original $4 \mathrm{GHz}$ rate. When the TFB is active each sub-band is processed in one correlator plane thus enhancing the number of correlators. Each sub-band can also be processed by several planes to improve the spectral resolution as sometimes required for 'connected array' observations. With 32 planes and 4 correlator cards per plane a total of 512 correlator cards are needed for the full 4 baseband pairs in the system. With 64 correlator chips assembled per card and 4096 2-bit multipliers (lags) per chip the total number of hardware correlators is huge.

\subsection{Spectral modes}

Many different spectral resolutions across different bandwidths are available as well as different polarization modes (1, 2 or 4 polarization products) or sensitivity options (Nyquist or double Nyquist sampling, 2-bit or 4-bit correlation). The pure XF mode (where the TFB cards are bypassed) provides coarse spectral resolutions (from about 8 to $30 \mathrm{MHz}$ ) while much finer spectral resolutions (around $1 \mathrm{MHz}$ down to a few $\mathrm{kHz}$ ) is available with the digital filtering system (frequency division mode). The latter is the adopted mode for ALMA phasing (see Section 3) primarily because implementing and monitoring phase corrections in the TFB subsystem is relatively easy with the existing hardware and because processing $62.5 \mathrm{MHz}$ slices is consistent with the existing fiber transport technology and new VLBI recorders.

\subsection{Status}

The 64-antenna correlator is organized by quadrants which have all been delivered to the Array Operations Site (AOS) at an elevation of $5050 \mathrm{~m}$. After extensive testing at the integration center the first, second, third and fourth quadrants have been transported to Chile and installed at the AOS in 2008, 2009, 2010 and 2012, respectively. At the time of this meeting, testing of the full 4-quadrant configuration is nearly complete. It allows us to process up to 64 antennas in the array and thus doubles the processing capacity which has been in use during the early science program while the ALMA construction continued.

\section{Phasing the ALMA array}

The electronics required for VLBI projects were not included in the initial ALMA construction project. However, no technical options were made which would have compromised the ability to phase up the ALMA array or a subset of its antennas. Following intensive 
discussions, a large group of engineers and scientists has proposed a global functional block diagram to phase up the ALMA array which includes new software/firmware development. This diagram can be found in [2]. The correlator is used in the frequency division mode (32 subbands per $2 \mathrm{GHz}$ baseband). The phase corrections to form the coherent sum are applied in the phase registers of the TFB cards. The phase of each sub-band must be appropriately calibrated. The frequency of the calibration session is to be determined and depends on many operational conditions such as the observing frequency, array (or sub-array) configurations, site weather, etc. The phases will be accurately aligned via the digital synthesizers of each TFB card. In addition to these phase shifts, gain corrections are needed during VLBI observations. These can also be applied in the TFB card. The phase and gain corrections do not require new hardware development. On the other hand, new software development is required to close the "phasing loop'. The $62.5 \mathrm{MHz}$ wide phase-corrected TFB sub-band signals are passed to the correlator cards which already have the logic required to sum the antennas which have been preselected for VLBI observations ( 1 to 64 antennas). The existing correlator cards provide for each polarization a scaled sum of 2-bit signals from up to 64 antennas. This later needs to be properly formatted for VLBI recording (VDIF).

In the following sub-sections, we focus our attention on the additional hardware required in the correlator and how it will be validated. We do not discuss here all other questions in relation with the operational VLBI mode (hydrogen maser, $1 \mathrm{pps}$ distribution, recording etc.) and do not address anything related to the complex software development.

\subsection{Phasing interface card}

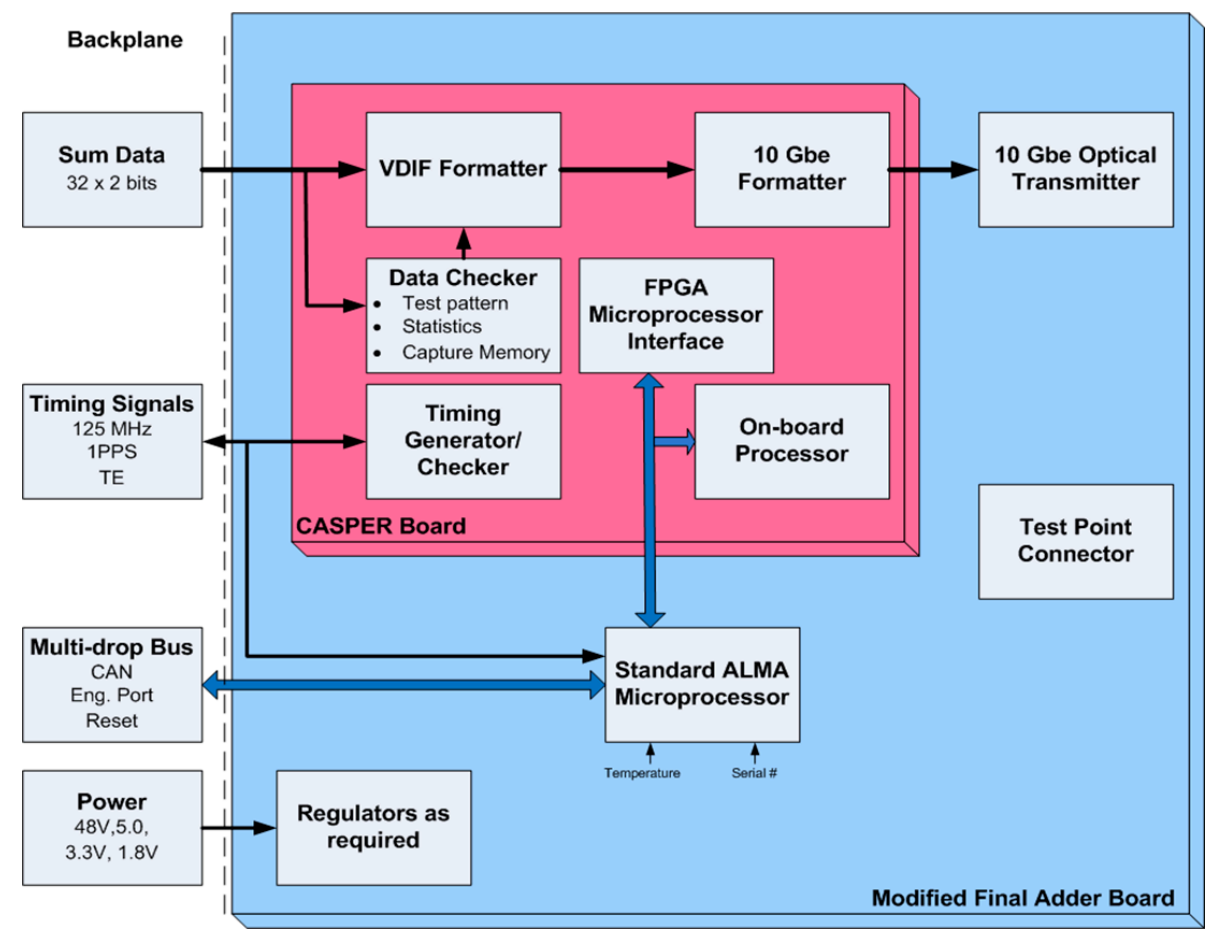

Figure 1: Functional block diagram of the Phasing Interface Card (PIC) processing 32 sub-bands summed data loaded from the correlator rack backplane to deliver packetized data to the optical transmitter. 
The phased summed signals must be connected to a new Phasing Interface Card (PIC) which will aggregate the 32 coherent sums. It is planned to have 2 such cards per quadrant. The slots needed to insert the 8 PICs in the correlator racks are identified and there is enough power in the system to supply these cards; the cooling in the correlator racks is adequate to accomodate 8 more cards. Some details of the PIC are shown in Fig. 1. The design consists of two cards which are mated together. One of these, labeled "Modified Final Adder Board", preserves the look and feel of the correlator, mainly by providing the microprocessor interface common to other cards in the correlator and using a form factor that allows it to fit into existing slots in the correlator. The second board, which will be mounted as a daughter card on the first, is a commercially available board, the "ROACH-2", produced by the CASPER collaboration". This board provides a large FPGA to process data. The collaboration provides a set of tools for developing applications for the board. In our application, the $32 \times 2$-bit sum data at the PIC input are formatted in the VDIF VLBI format, packetized into 10GbE packets and sent to the fiber multiplexer for fiber transport from the AOS to the lower 2900-m Operations Support Facility (OSF) where the VLBI recorders are installed; only one fiber will be needed for the AOS to OSF transport with the proposed multiplexer/demultiplexer.

\subsection{PIC test fixture and PIC card status}

Two ALMA data simulator cards and existing ALMA correlator cards (TFB and station cards, correlator interface and correlator cards etc.) will be connected to a PIC card to form a test fixture simulating the full correlator system (Fig. 2).

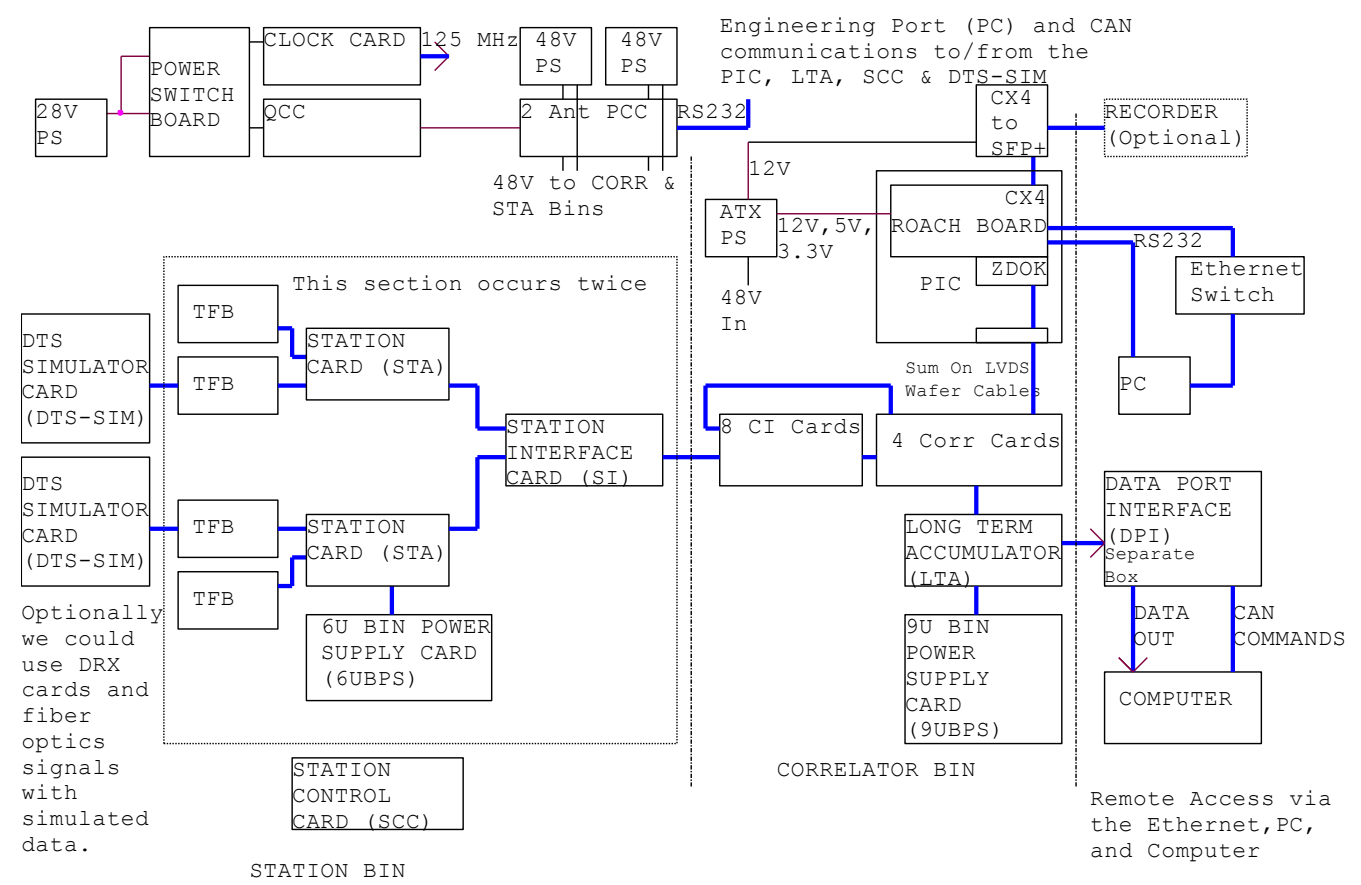

Figure 2: Main elements of the PIC test fixture.

\footnotetext{
${ }^{2}$ Collaboration for Astronomy Signal Processing and Electronics Research, https://casper.berkeley.edu/
} 
This test equipment will allow checking the PIC functionality. With feedback to the correlator interface card (see Fig. 2), the phasing efficiency can be tested by correlating the summed antenna signals against one reference antenna. In the operational system some delay adjustment will be required for the reference antenna; the procedure to perform this adjustment can also be tested with the PIC test fixture. In addition, if a real VLBI recorder can be connected to the output of the PIC we then have in the laboratory a rather complete 'VLBI mode simulator'. This will help to minimize the testing period with the operational system in the field.

The PIC card and test fixture are being developed and assembled by the NRAO team in Charlottesville. The layout of the PIC card will be complete shortly after the preliminary design review of the ALMA Phasing Project (review scheduled on 1-2 November 2012).

\section{Concluding remarks}

Phasing up the ALMA array will undoubtedly enhance the ALMA science objectives. New (sub)mm VLBI observations of black holes in our Galaxy or AGNs and, for instance, observations of galactic/extragalactic cosmic masers will become possible with unprecedented sensitivity. The phased ALMA array, as a single telescope, will also allow mm observations of pulsars. The basic equipment needed to implement the ALMA phasing system is in place in the correlator original design - TFB cards can align the phase differences measured in the 32 subbands of each baseband; the correlator cards provide the sum of a selectable set of antennas. The summed data are transmitted to a new interface card (PIC) whose design is nearly complete. This card will format the data for fiber transmission to the VLBI recorders. An associated PIC test fixture is being assembled so that much testing of the PIC and VLBI modes will be possible in the laboratory without disturbing ALMA current operation.

\section{References}

[1] A. Baudry, The ALMA radio telescope, in Proceedings of Science ( $2^{\text {nd }}$ MCCT-SKADS) 002 (Siguenza, Spain, 2008) http://pos.sissa.it/archive/conferences/065/002/2nd\%20MCCTSKADS 002.pdf

[2] S. Doeleman, ALMA Beamforming Project, in ALMA Phasing Kickoff Meeting (Haystack, December 2011) http://eventhorizontelescope.org/collaborators/meetings/alma kickoff/docs/alma overviewDoeleman.pdf

[3] H. Falcke, R. Laing, L. Testi, A. Zensus, mm-wave VLBI with ALMA and Radio Telescopes around the World, The Messenger, vol 149, p. 60 (2012)

[4] A. Baudry, R. Lacasse, R. Escoffier, et al., Performance Highlights of the ALMA Correlators, Proc. of SPIE, vol. 8452, id. 845217 (SPIE conference, Amsterdam, July 2012) 